\title{
Essentially Nonoscillatory (ENO) Reconstructions via Extrapolation
}

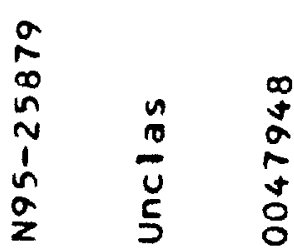

$\underbrace{-1}_{0}$

Ambady Suresh

NYMA, Inc.

Engineering Services Division

Brook Park, Ohio

and

Philip C.E. Jorgenson

National Aeronautics and Space Administration

Lewis Research Center

Cleveland, Ohio

Prepared for the

33rd Aerospace Sciences Meeting and Exhibit

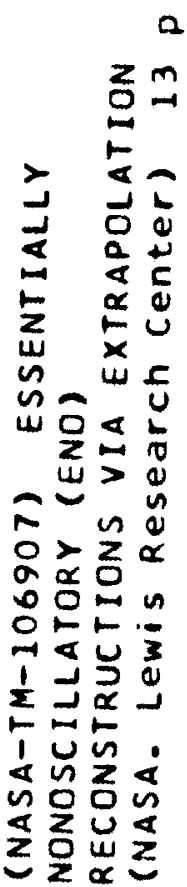
sponsored by the American Institute of Aeronautics and Astronautics Reno, Nevada, January 9-12, 1995

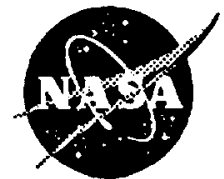

National Aeronautics and Space Administration 


\title{
ESSENTIALLY NONOSCILLATORY (ENO) RECONSTRUCTIONS VIA EXTRAPOLATION
}

\author{
Ambady Suresh $†$ \\ NYMA Inc., \\ NASA Lewis Research Center, Cleveland, Ohio 44135 \\ and \\ Philip C. E. Jorgenson ${ }^{\dagger}$ \\ Computational Fluid Dynamics Branch \\ NASA Lewis Research Center, Cleveland, Ohio 44135
}

\begin{abstract}
In this paper, the algorithm for determining the stencil of a one-dimensional Essentially Nonoscillatory (ENO) reconstruction scheme on a uniform grid is reinterpreted as being based on extrapolation. This view leads to another extension of ENO reconstruction schemes to two-dimensional unstructured triangular meshes. The key idea here is to select several cells of the stencil in one step based on extrapolation, rather than one cell at a time. Numerical experiments confirm that the new scheme yields sharp nonoscillatory reconstructions and that it is about five times faster than previous schemes.
\end{abstract}

\section{Introduction}

ENO (Essentially Nonoscillatory) schemes developed by Harten, Osher, Shu and others $[1,2,3,4]$ are a class of higher order numerical schemes for solving hyperbolic partial differential equations. These equations frequently admit solutions in which some smooth structure is interspersed with discontinuities. ENO schemes are designed so as to achieve a high order of accuracy in the smooth regions and at the same time avoid spurious oscillations at discontinuities. In such cases, conventional fixed stencil schemes either damp out the smooth structure while obtaining a monotone shock profile or capture the smooth structure and incur spurious oscillations near the shock.

The key feature of ENO schemes is the use of an adaptive stencil in the reconstruction step, i.e. in constructing point values of $u(x)$ from the set of cell

$\dagger$ Member, AIAA. averages $\bar{u}_{i}$. For each cell, a searching algorithm determines which stencil of neighboring points has the smoothest data and $u(x)$ is then reconstructed using data only from this stencil. The variable stencil has the effect of avoiding $O(1)$ spurious oscillations near discontinuities.

With unstructured grids offering greater geometric flexibility and ease of adaptation, many efforts $[5,6,7,8]$ have been made to extend ENO schemes to unstructured grids. Harten and Chakravarthy [5] describe a cell centered extension of the finite volume ENO scheme to triangulated unstructured grids along with details of implementation although no numerical results are presented. Abgrall $[6,7]$ presents an ENO cell vertex scheme where the control volumes are the cells of the dual mesh and presents results for $1 \mathrm{D}$ convection and gas dynamics.

In this paper, we first reinterpret the basic one dimensional ENO scheme on a uniform grid as a scheme based upon extrapolation. Based on this point of view, we present another extension of ENO schemes to unstructured meshes. This new scheme has the advantage that it is more efficient than the scheme of Harten and Chakravarthy[5] and gives almost identical results on fine enough meshes. We consider only reconstruction in this paper. Implementation of this scheme in a flow solver will be the subject of future work.

\section{D ENO Reconstruction Revisited}

Consider $m-1$-th order ENO reconstruction of a function $u(x)$ using the primitive function technique (see [1] for details). Let the cell of interest be $\left[x_{j}, x_{j+1}\right]$ with cell average $\bar{u}_{j+1 / 2}$. The reconstruction will use the cell averages $\left[\bar{u}_{j+1 / 2-m+1}, \ldots \bar{u}_{j+1 / 2+m-1}\right]$. The primitive function of $u(x)$ is defined by

$$
H(x)=\int_{x_{0}}^{x} u(\xi) d \xi
$$


and is known at the cell faces up to an additive constant. An $m$-th order interpolant $Q(x)$ that interpolates $H(x)$ at $\left[x_{i}, \ldots x_{i+m}\right]$ is constructed next and the reconstruction, denoted by $v_{j+1 / 2}(x)$, is then obtained as

$$
v_{j+1 / 2}(x)=\frac{d Q}{d x}
$$

There are precisely $m$ choices for $i$ namely $[j-m+$ $1, \ldots . j]$. The nonoscillatory properties of the reconstruction result from choosing $i$ so that $\left[x_{i}, \ldots x_{i+m}\right]$ is the smoothest possible region around the cell $\left[x_{j}, x_{j+1}\right]$. One way of doing this is by a recursive procedure, as described below.

We begin with $i=j$, the linear interpolant for $Q(x)$ for which the stencil is $\left[x_{j}, x_{j+1}\right]$, and keep adding cells until we have the required number. Assume that at some stage in this process the stencil is $\left[x_{i}, \ldots x_{i+p}\right]$. At the next step, the choice is between the two stencils $\left[x_{i}, \ldots x_{i+p+1}\right]$ or $\left[x_{i-1}, \ldots x_{i+p}\right]$. If $H\left[x_{i}, \ldots x_{i+p+1}\right]$ is less than $H\left[x_{i-1}, \ldots x_{i+p}\right]$, where $H[.$.$] denotes the divided$ differences of $H(x)$, the former stencil is picked, otherwise the latter. In current practice, it is customary to weight the two divided differences being compared so as to favor stencil movement towards the centered stencil. However, this is not crucial to the equivalence we wish to establish below and thus has been dropped for clarity.

Let us now consider a different method of stencil selection. We begin as before and assume that at some stage in this process the stencil is $\left[x_{i}, \ldots x_{i+p}\right]$. We reconstruct $u(x)$ based on this stencil and denote this reconstruction by $v^{p}(x)$. Now, we extrapolate $v^{p}(x)$ to the neighboring cell $\left[x_{i+p}, x_{i+p+1}\right]$, evaluate the cell average of $v^{p}(x)$ over this cell, and subtract from this the true cell average of this cell. Let us denote this residual as $R^{+}$. The same thing can be done for the other neighboring cell $\left[x_{i-1}, x_{i}\right]$ and we end up with another residual which we denote as $R^{-}$. If $\left|R^{+}\right|<\left|R^{-}\right|$, we choose $\left[x_{i}, \ldots x_{i+p+1}\right]$ for the stencil and proceed to the next step. If not, the stencil chosen is $\left[x_{i-1}, \ldots x_{i+p}\right]$.

Since the reconstruction at any stage is the derivative of a primitive function, the values of the residuals can be calculated as

$$
\begin{aligned}
& R^{+}=H\left[x_{i}, \ldots, x_{i+p+1}\right] \prod_{k=0}^{p-1}\left(x_{i+p+1}-x_{i+k}\right) \\
& R^{-}=H\left[x_{i-1}, \ldots, x_{i+p}\right] \prod_{k=0}^{p-1}\left(x_{i+k+1}-x_{i-1}\right)
\end{aligned}
$$

It follows that for a uniform grid this method of stencil selection is identical to the standard ENO method via divided differences of $H$ since the two products in Eqs. (3) and (4) are the same. As in standard ENO, the residuals can be appropriately weighted so as to favor stencil movement towards the centered stencil. In smooth regions, the residuals are $O\left(\Delta x^{p}\right)$ while if the extrapolated cell contains a jump discontinuity the residual is $O(1)$, entirely analogous to scaled divided differences. For nonuniform grids in one dimension, the two methods differ because of different weighting factors on the divided differences and it is not clear to us which is the better choice. Limited numerical experiments with the new method yield solutions that are similar to that from standard ENO.

The extrapolation method outlined above is reminiscent of subcell resolution introduced by Harten[9]. There, the extrapolation is performed to locate the discontinuity within a cell. Unlike subcell resolution, the extrapolation method can be readily extended to multidimensions as we show below. In addition, ENO interpolation can also be viewed as being based on extrapolation. In this case, the extrapolated point value is compared to the true point value, their difference being the appropriate residual.

In what follows below, we apply this extrapolation idea to the problem of ENO reconstruction on a twodimensional unstructured mesh.

\section{ENO Reconstruction on Unstructured Grids.}

An unstructured triangular mesh is characterised by a set of vertices $\left(x_{i}, y_{i}\right)$, where $i=1, . . n v$ and a set of connection coefficients $c(i, j)$ where $i=1, n c$ and $j=$ 1,3. $n c$ is the number of cells or triangles and $n v$ the number of vertices. For the $i$-th. triangle, $c(i, j)$ specify the labels of its vertices. A given set of vertices may be connected in many ways to form an unstructured grid. A systematic way of connecting a given set of points is the so called Delaunay triangulation which has several desirable properties.

We will consider only cell centered schemes in this work so that the control volumes or cells will be the triangles themselves. The reconstruction problem can be stated as follows: Given the cell averages of a piecewise smooth function $u(x)$, reconstruct the function within a particular cell in a nonoscillatory manner to within a specified order of accuracy. For some theoretical results on this problem, the reader is referred to Abgrall[6,7].

The first step is to consider the case where $u(x)$ is globally smooth. Let $C_{i}$ be the cell of interest whose centroid is chosen as the origin of the coordinates $(x, y)$. For an $m$-th order reconstruction we seek the recon- 
structed function as

$$
v(x, y)=\sum_{l=0}^{m} \sum_{j+k=l} a_{j, k} x^{j} y^{k}
$$

Since there are $(m+1)(m+2) / 2$ unknowns, it is clear that we need a stencil of at least that many cells near $C_{i}$. For conservation, one of these cells must be $C_{i}$. How these cells are selected lies at the heart of the ENO reconstruction procedure and will be discussed below. For now, assume a stencil of cells with cell indices $J_{q}(i)$, where $q=1, \ldots(m+1)(m+2) / 2$ have been found. The linear system for determining $a_{j, k}$ can be written as

$$
\sum_{l=0}^{m} \sum_{j+k=l} a_{j, k}<x^{j} y^{k}>_{q}=\bar{u}_{q}
$$

where $q=1, \ldots(m+1)(m+2) / 2$ and

$$
<x^{j} y^{k}>_{q}=\frac{1}{\left|C_{J_{q}}\right|} \iint_{C_{J_{q}}} x^{j} y^{k} d x d y
$$

There is no guarantee that the linear system (6) will be nonsingular. but when it is, the solution of it yields the required reconstruction. It is clear, however, that the matrix will be singular only for special cases.

We now describe a method of selecting the stencil due to Harten and Chakravarthy[5] which we shall refer to as ENOHC. We begin with the single triangle $C_{i}$ for which the zero-th order reconstruction is $a_{0,0}=\bar{u}_{i}$ or

$$
v(x)=\bar{u}_{i}
$$

Let us denote by $J_{p}(i)$ a stencil of $p$ cells at the $p$-th stage of the stencil selection process where

$$
J_{p}(i)=\left\{i_{1}, i_{2}, \ldots i_{p}\right\}
$$

These $p$ triangles can be used to fit $p$ terms of the expansion(5) so that associated with this stencil are $p$ values of $a_{j, k}$. While these $p$ terms can be taken in any order, it is natural to take them in the lexicographic order, namely, $1, x, y, x^{2}, x y, y^{2}, \ldots$ Let $J_{p}^{*}$ denote cells not in $J_{p}(i)$ that share a common side with cells in $J_{p}(i)$. In the next step we add to $J_{p}(i)$ any one triangle from $J_{p}^{*}$ and fit the $p+1$ terms in the expansion (5) using the $p+1$ triangles. We do this for all triangles in $J_{p}^{*}$ and pick the one that minimizes the sum of the absolute values of the $p+1$ coefficients $a$. The stencil now contains $p+1$ triangles and $p+1$ values of $a_{j, k}$ and we proceed to the next step. When $p=(m+1)(m+2) / 2-1$ the process is complete and we have the required $m$-th order ENO reconstruction.

The cost of adding one cell to the stencil can be roughly estimated as follows. For every element of $J_{p}^{*}$, calculating the norm of the coefficients a involves calculating $2 p+1$ quadratures (the additional row and column of the linear system (6)) one $p+1 \times p+1$ matrix inversion and a norm calculation. Neglecting the cost of finding the minimal element, the approximate cost of adding one cell to the stencil may be written as

$$
\left|J_{p}^{*}\right|((2 p+1) Q+M(p+1)+N(p+1))
$$

where $Q$ is the cost of a quadrature, $M(n)$ is the cost of inverting an $n \times n$ matrix and $N(n)$ is the cost of a norm calculation of $n$ terms.

This method seems to be a logical extension of the standard ENO scheme in one dimension. Numerical tests that we have performed with the method (to be presented below) show that sharp nonoscillatory reconstructions are obtained away from the boundary. A curious feature of the scheme is that the stencil chosen and hence the reconstruction obtained are not coordinate invariant, which is due to the fact that terms are fitted one by one. The main drawback of the scheme is its expense, especially at higher orders. This may be traced to the fact that the number of triangles required is proportional to $m^{2}$ and triangles are added one by one. To go from an $m$-th order reconstruction to $m+1$-th order requires the addition of $m+2$ cells to the stencil, each of which is added in a separate step. In the next section, we introduce a simplification that will allow us to choose many cells together resulting in a more efficient algorithm.

\section{Stencil Selection via Extrapolation}

As before, we begin with the single triangle $C_{i}$ and the zero-th order reconstruction given by Eq.(8). Assume we have an $l$-th order reconstruction for the cell of interest. This means we have a stencil $J_{p}(i)$ of $p=(l+1)(l+2) / 2$ cells and a reconstruction of the form

$$
v_{l}(x)=\sum_{q=0}^{l} \sum_{j+k=q} a_{j, k} x^{j} y^{k}
$$

As before, we have the set of neighbors to this stencil $J_{p}^{*}$. For every candidate cell in $q \in J_{p}^{*}$ we compute the residual given by

$$
R_{q}=\bar{u}_{q}-\frac{1}{\left|C_{q}\right|} \iint_{C_{q}} v_{l}(x, y) d x d y
$$

If the cell $C_{q}$ and all cells in the stencil $J_{p}(i)$ lie in a smooth region, the residual is $O\left(h^{l+1}\right)$ where $h$ is some representative cell size. If not, the residual is $O(1)$. Hence, we compute this residual for all candidate triangles and pick those $l+2$ triangles that have the 
least value of the residual. Once we select the $l+2$ additional triangles, we perform a linear reconstruction problem to obtain the $l+1$-th order reconstruction. Then we proceed to the next stage until when $l=m$ we have the desired reconstruction.

The key difference between ENOHC and this method is that we select all $l+2$ triangles in one step. This is also the main problem with this method which works best when every cell has a large number of neighbors. In many cases, especially for the lower orders, there are not enough triangles. For example, while going from the zero-th order reconstruction to a first order reconstruction, we have to select 2 triangles from the 3 neighbors. This is usually not enough of a choice and the reconstruction is often oscillatory. Numerical tests suggest two further modifications which are necessary for a robust reconstruction algorithm. The first is we have found it necessary to limit the order of the reconstruction based on the magnitude of $a_{j, k}$. Specifically, we compute the norm

$$
N(l)=\frac{1}{(l+1)(l+2) / 2} \sum_{q=0}^{l} \sum_{j+k=q}\left|a_{j, k}\right|
$$

and if $N(l)>1 / h$ we terminate the reconstruction at that stage. Unlike ENOHC, this has the effect of reducing the order of the reconstruction near discontinuities, in the same spirit as Suresh[10]. There does not seem to be a clear choice for the mesh refinement parameter $h$ for unstructured meshes. In our numerical experiments, $h$ was chosen to be the minimum of the circumcircle radii.

The second modification is based on the detectability of the residuals. As mentioned above, the residual for a cell in the smooth region is $O\left(h^{l+1}\right)$ while for a cell containing a discontinuity it is $O(1)$. Thus detection of the "bad" cells is most difficult at the lower values of 1. On the other hand, ENOHC is relatively inexpensive at the lower orders. Hence, the first few stages can be obtained using ENOHC and the latter stages obtained using extrapolation. Thus the second modification that we have used is to obtain a first order reconstruction using ENOHC and use the extrapolation procedure from then on. In numerical tests below, we shall refer to the scheme described above as ENOEX.

We remark that a scheme in between ENOHC and ENOEX can also be constructed which like ENOHC adds cells one by one but uses extrapolation to select the cells. Here, at the $p$-th stage of stencil selection, we compute the residual (12) for all cells in $J_{p}^{*}$ and pick a single cell that has the least residual. This triangle is added to $J_{p}$ and the associated linear system solved to get the new $p+1$ values of $a_{j, k}$ and we proceed to the next stage. The effort involved in going from a stencil of $p$ cells to $p+1$ cells is one quadrature for every element of $J_{p}^{*}$ and one matrix inversion or

$$
\left|J_{p}^{*}\right| Q+M(p+1)
$$

This is still a substantial saving over Eq.(10) although much more expensive than ENOEX. We do not consider this intermediate scheme any further.

\section{Numerical Experiments}

We describe below several numerical tests performed on the new ENOEX reconstruction algorithm. The unstructured grids used for these purposes were generated by applying random perturbations to the vertices of a structured grid and then applying the BowyerWatson Delaunay triangulation algorithm to the result. Typical grids are shown in Fig. 1 and Fig. 9, where the domain of reconstruction is $[-1,1] \times[-1,1]$. For the coarse grid (Fig. 1) a structured grid of $40 \times 40$ was used and the magnitude of the random perturbation was set to $\pm 40 \%$ of the structured mesh size (i.e. 0.05 ). For the fine grid, a structured grid of $80 \times 80$ was used with the same relative magnitude of the perturbations. The perturbations are set to zero on the boundary. The cell size parameter in ENOEX was chosen to be the minimum of the circumcircle radii over the whole grid. This is obtained from the Bowyer-Watson algorithm and fixed apriori in the computations presented below.

In studying the accuracy of the reconstructions, contour plots of functions on unstructured meshes can be made with presently available software tools. However, getting one-dimensional line plots of the function along curves is often difficult and involves further interpolation. For this reason, we have found it convenient to perform the reconstruction on to the nodes of a suitably chosen structured mesh. From the structured mesh values it is quite easy to study one-dimensional line plots along the coordinate directions. The structured mesh is chosen so that there is roughly one node per cell and is $41 \times 41$ for the coarse grid and $81 \times 81$ for the fine mesh.

\section{Reconstruction of a smooth function}

The first tests are performed on smooth functions and serve as a convenient debugging mechanism. We reconstructed the smooth function

$$
u(x, y)=\sin (\pi(x+y))
$$

on the two unstructured meshes shown in Figs. 1 and 9 respectively using ENOEX4 (fourth order ENOEX 
reconstruction). The errors in the max-norm and computed order of accuracy are shown in Table-1. These errors were computed at the centroid of each cell. Since there is no well defined mesh refinement parameter, we have calculated the order of accuracy based on four different parameters, namely the maximum and minimum circumcircle radii and the square root of the maximum and minimum cell areas. The numerical order of accuracy varies from 6.28 to 3.55 , depending on the mesh refinement parameter used. In theory, all should approach 5 on fine enough meshes.

\section{Reconstruction of a piecewise smooth function}

Since we deal only with the reconstruction problem, the initial function to be reconstructed has to be sufficiently general. A suitable function has been constructed by Abgrall [6] which has several curved discontinuities and smooth structures. The function (which is slightly different from that in Abgrall[6]) is defined as follows: If $\phi$ is any angle and $r=-(y-x \tan (\phi) / 3$ then $f_{\phi}(x, y)$ is defined as:

$$
\left\{\begin{array}{l}
\text { if } \quad r \leq-1 / 3, \quad \mathbf{f}_{\phi}(x, y)=-r \quad \sin \left(\pi r^{2} / 2\right), \\
\text { if } \quad r \geq 1 / 3, \quad \mathbf{f}_{\phi}(x, y)=2 r-\sin (3 \pi r) / 6, \\
\text { if } \quad|r|<1 / 3, \quad \mathbf{f}_{\phi}(x, y)=|\sin (2 \pi r)|
\end{array}\right\}
$$

and the function to be reconstructed $u(x, y)$ is

$$
\begin{aligned}
& u(x, y)=f_{\sqrt{\pi / 2}}(x, y) \quad \text { if } \quad \mathrm{x} \leq \cos (\pi \mathrm{y} / 2) \\
& u(x, y)=f_{-\sqrt{\pi / 2}}(x, y)+\cos (2 \pi y) \\
& \text { if } \quad \mathrm{x}>\cos (\pi \mathrm{y} / 2)
\end{aligned}
$$

The cell averages of this function over each cell are computed by using an adaptive quadrature routine(from the IMSL library), where in most cells, the estimate of absolute error is machine zero. However, for cells near discontinuities, the absolute errors are larger, with the largest error being $O\left(10^{-6}\right)$.

Figures 3 and 4 show the results of reconstruction using ENOHC4 and ENOEX4 (fourth order reconstructions) respectively on the coarse mesh shown in Figure 1. Note that this reconstruction corresponds to a fifth order scheme for convection or gas dynamics. Figure 2 is a contour plot of the exact solution. It can be seen that the results of ENOEX 4 are comparable to ENOHC4 although the latter has slightly better resolution at discontinuities. Figures $5-8$ show line plots of the reconstructions at various $x$ and $y$ sections of the domain. For the most part, the reconstructions are similar with ENOEX showing a slightly larger undershoot in Figure 7. Figures 11 and 12 show corresponding results for the fine mesh shown in Figure 9. In this case, the two reconstructions are almost identical with the only difference being at about $(-0.4,-0.9)$ where ENOEX4 is a bit noisy.

The CPU time comparisons are presented in Table 2. Since a lot of time in these runs is spent in computing the intersections with the structured grid and in the computation of the cell averages, these times were not included in the run times listed in Table-2. It can be seen that ENOEX4 scheme is about five times faster than ENOHC4. These comparisons are meant to serve only as a guide as they depend on the efficiency of programming. Furthermore, the gap narrows for lower orders being identical for $m=1$.

\section{Conclusions and Remarks}

In this paper, we have proposed a new essentially nonoscillatory (ENO) reconstruction procedure on twodimensional unstructured meshes. At the heart of the method is a reinterpretation of standard ENO reconstructions as being based on extrapolation. Numerical experiments confirm that the reconstruction is indeed nonoscillatory and that the new fourth order scheme is about five times faster than earlier schemes.

We view ENOEX as a first step towards faster ENO schemes on unstructured meshes. Many issues need to be investigated further. Weighting of the central stencil, which is important for stability considerations, can be accomplished in several ways, with and without some notion of distance from the cell of interest. Better choices of the cell size parameter may exist. These questions will be explored further in future work.

\section{Acknowledgements}

The authors would like to thank David Watson of the University of Western Australia for the Delaunay triangulation algorithm. We would also like to thank William J. Coirier, H. T. Huynh and Jaap J. W. Van Der Vegt for several interesting discussions. This work was supported by NASA Lewis Research Center under Contract NAS3-25266 with Dr. D. R. Reddy as monitor.

\section{References}

1. A. Harten, B. Engquist, S. Osher, S. R. Chakravarthy, "Uniformly High Order Accurate Essentially Non-Oscillatory Schemes, III," J. Comput. Phys., 71, 231-303 (1987).

2. A. Harten, S. Osher, "Uniformly High-Order Accurate Nonoscillatory Schemes -I, SIAM J. Num. Anal., Vol. 24, No. 2, 279-309, (1987). 
3. C.-W. Shu, S. Osher, "Efficient Implementation of Essentially Non-oscillatory Shock Capturing Schemes," J. Comput. Phys., 77, 439-471, (1988).

4. C.-W. Shu, S. Osher, "Efficient Implementation of Essentially Non-oscillatory Shock Capturing Schemes, II," J. Comput. Phys., 83, $32-78$ (1989).

5. A. Harten, S. R. Chakravarthy, "Multidimensional ENO Schemes for General Geometries," ICASE Report No. 91-76, Hampton, Virginia, (1991).

6. R. Abgrall, "Design of an Essentially Nonoscillatory Reconstruction Procedure on Finite-Element Type Meshes," ICASE Report No. 91-84, Hampton, Virginia, (1991).

7. R. Abgrall, "On Essentially Nonoscillatory Schemes on Unstructured Meshes: Analysis and Implementation," ICASE Report No. 92-74, Hampton, Virginia, (1992).

8. P. Vankeirsblick, H. Deconinck "Higher Order Upwind Finite Volume Schemes with ENO-properties for General Unstructured Meshes.", Paper No. 7, AGARD R-787, May 1992, unpublished.

9. A. Harten, "ENO Schemes with Subcell Resolution," J. Comput. Phys., 83, 148-184, (1989).

10 A. Suresh, "ENO Schemes with Centered Stencils," AIAA 94-2296, 25th. AIAA Fluid Dynamics Conference, Colorado Springs, Colorado, June (1994). 
Table - 1

\begin{tabular}{|l|c|c|c|c|c|}
\hline \multicolumn{1}{|c|}{ Grid } & Max Error & $C_{\max }$ & $C_{\min }$ & $\sqrt{A_{\min }}$ & $\sqrt{A_{\max }}$ \\
\hline Coarse & $9.8698 \times 10^{-4}$ & 0.0542 & 0.0155 & 0.0100 & 0.0566 \\
\hline Fine & $3.9099 \times 10^{-5}$ & $2.8077(-2)$ & $6.2788(-3)$ & $6.0046(-3)$ & 0.0304 \\
\hline Order & - & 4.90 & 3.55 & 6.2870 & 5.1962 \\
\hline
\end{tabular}

Table 1: Reconstruction of the smooth function $\sin (\pi(x+y))$ on unstructured meshes. $C_{\min }$ and $C_{\max }$ are the minimum and maximum of the circumcircle radii and $A_{\min }$ and $A_{\max }$ are the minimum and maximum cell areas respectively.

Table - 2

\begin{tabular}{|l|c|c|}
\hline \multicolumn{3}{|c|}{ CPU Times (Ymp Seconds) } \\
\hline Grid & ENOEX4 & ENOHC4 \\
\hline Coarse & 21.694 & 124.252 \\
\hline Fine & 100.07 & 525.121 \\
\hline
\end{tabular}

Table 2: Comparison of CPU times for fourth order reconstruction using ENOHC and ENOEX. 


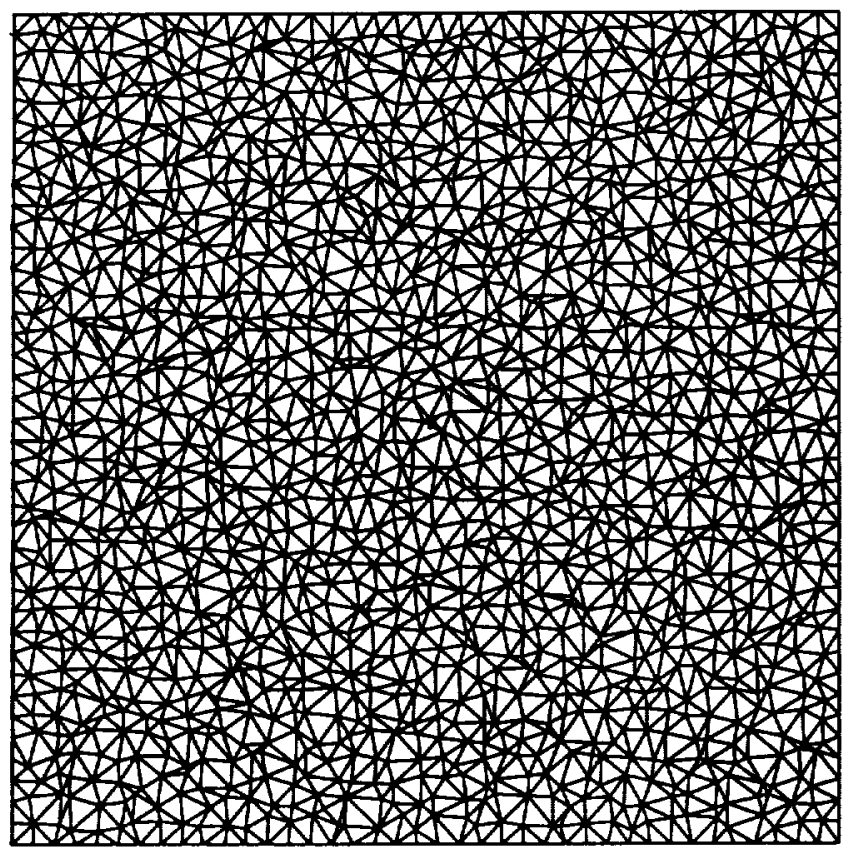

Figure 1: Unstructured Coarse Grid. 1600 vertices and 3024 triangles. Generated by random perturbations of a 40 X 40 uniform structured grid.

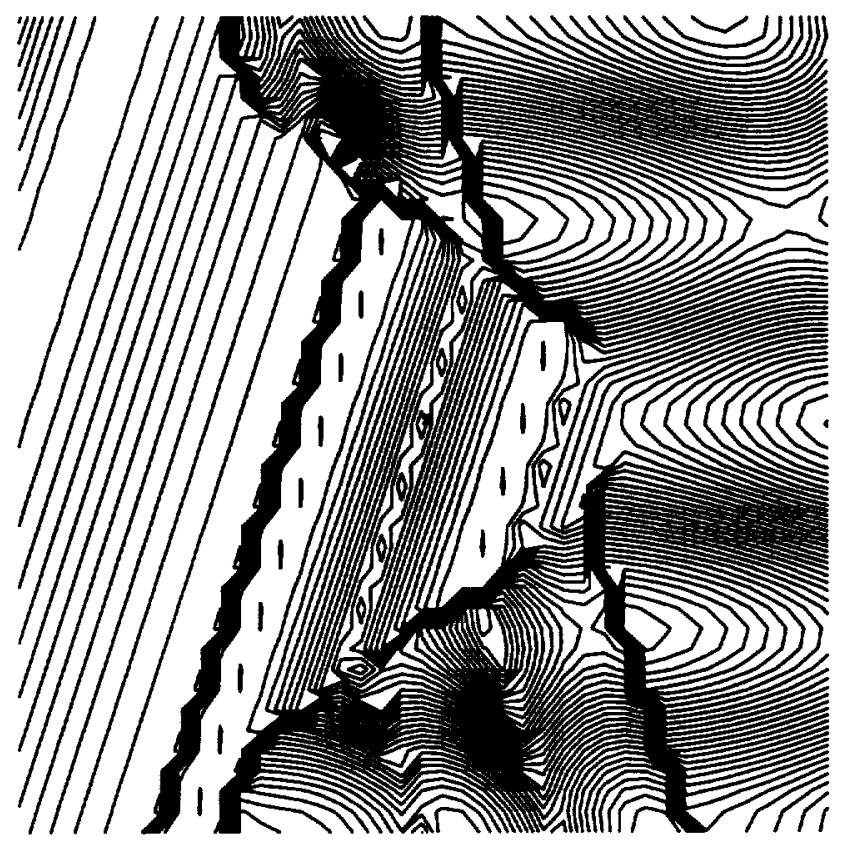

Figure 2: Exact solution on the coarse grid

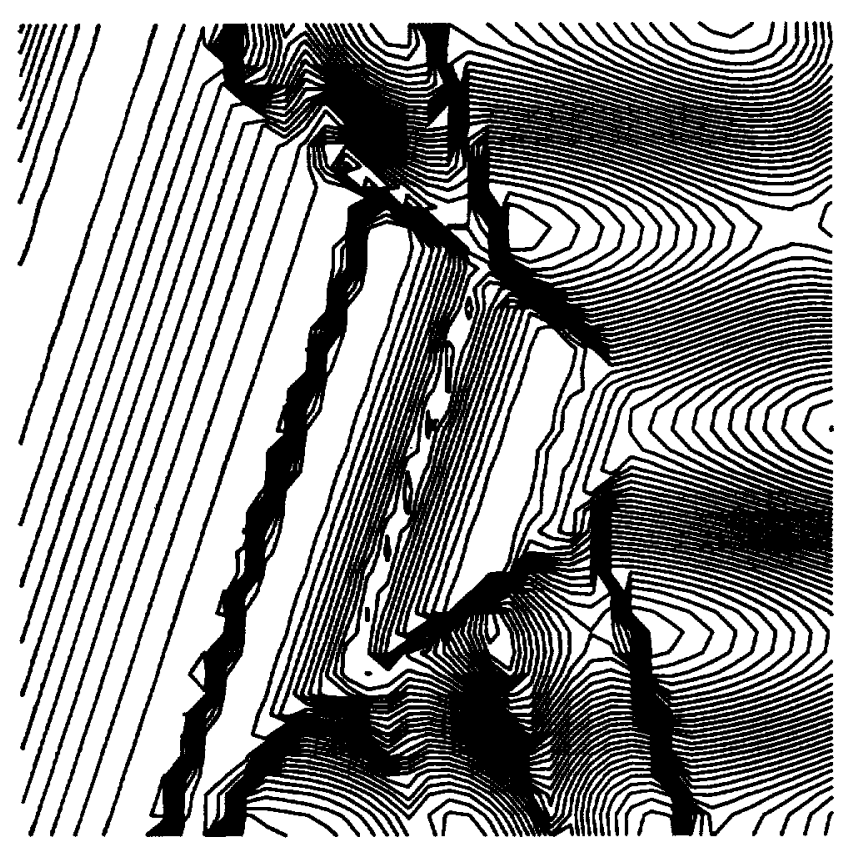

Figure 3: Fourth order ENOHC solution on the coarse grid

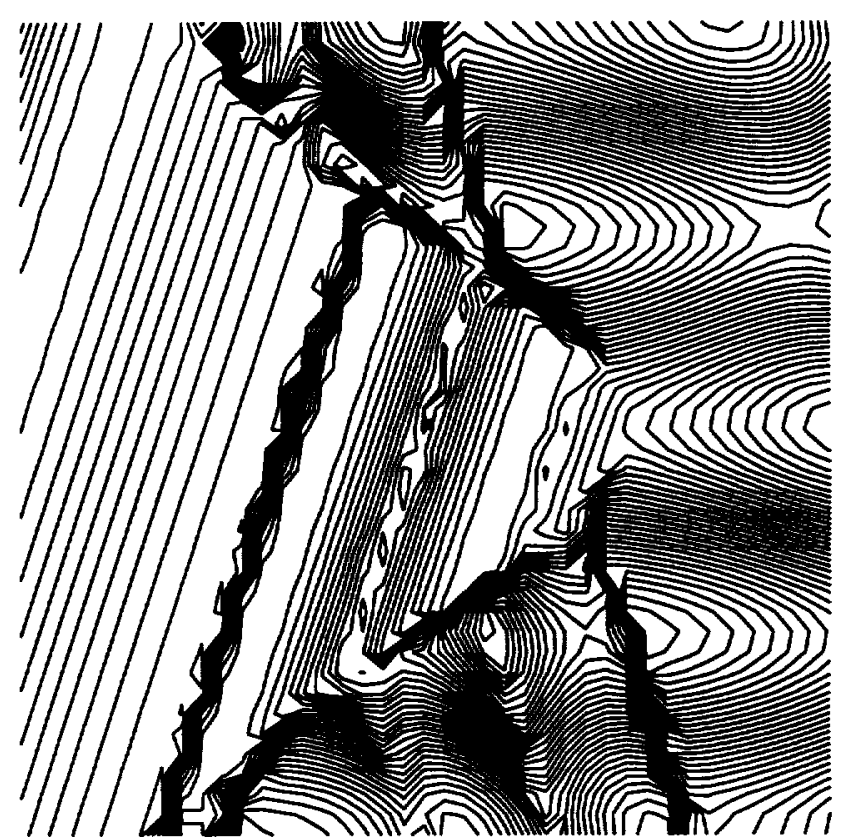

Figure 4: Fourth order ENOEX solution on the coarse grid 

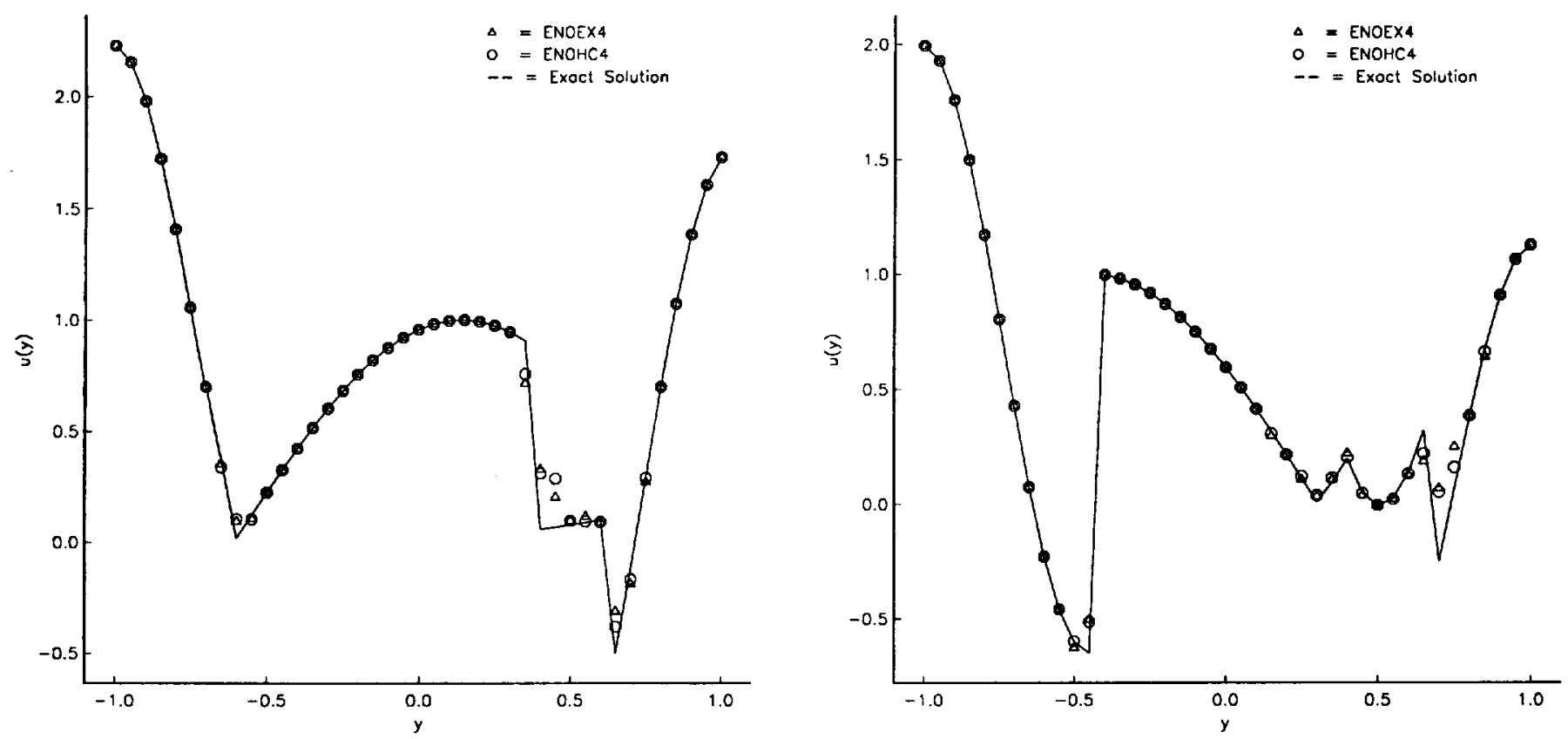

Figure 5: ENO Reconstructions on the line $\mathrm{x}=-0.2$. Coarse Figure 6: ENO Reconstructions on the line $\mathrm{x}=+0.1$. grid. Coarse grid.
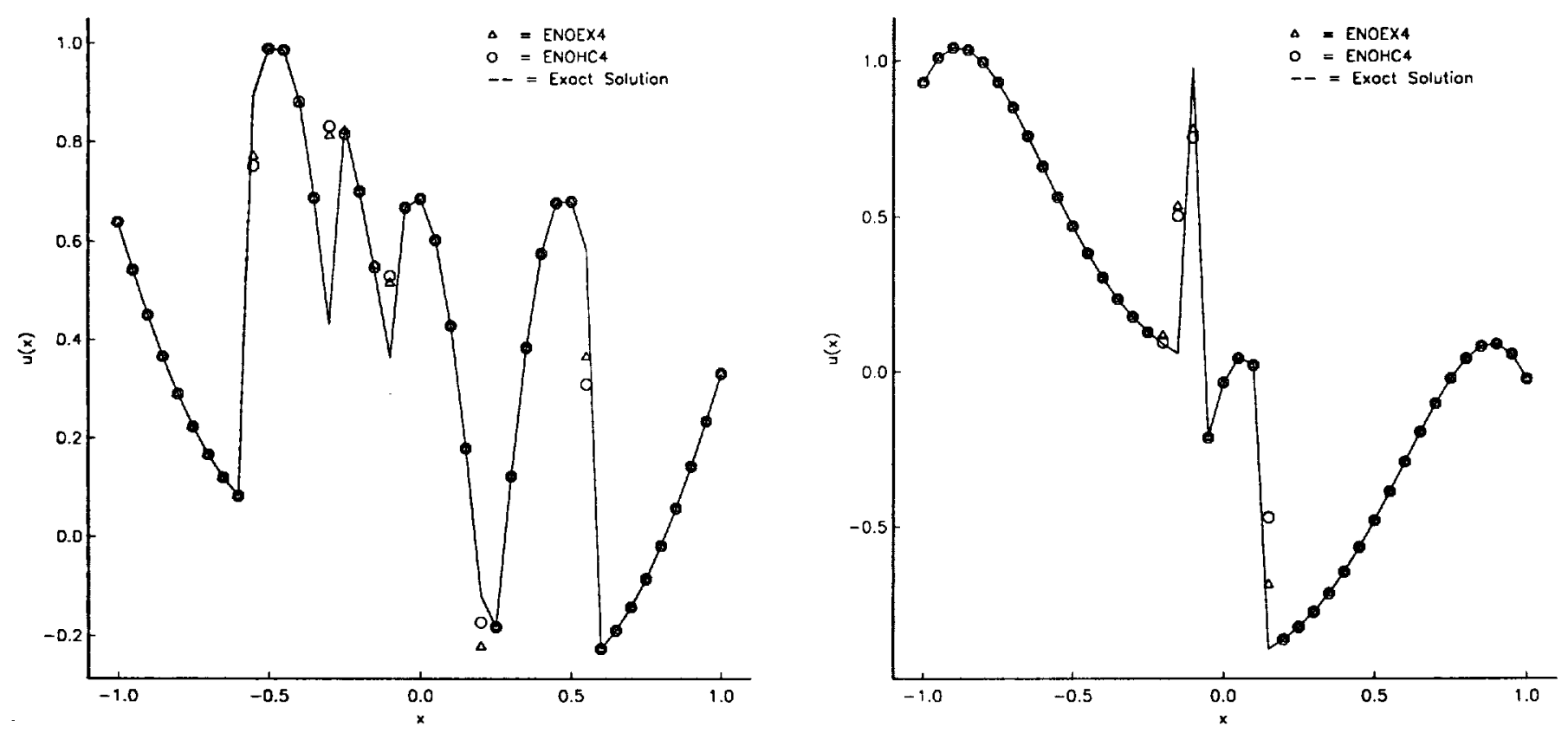

Figure 7: ENO Reconstructions on the line $y=-0.7$. Coarse grid.

Figure 8: ENO Reconstructions on the line $\mathrm{y}=0.55$. Coarse grid. 

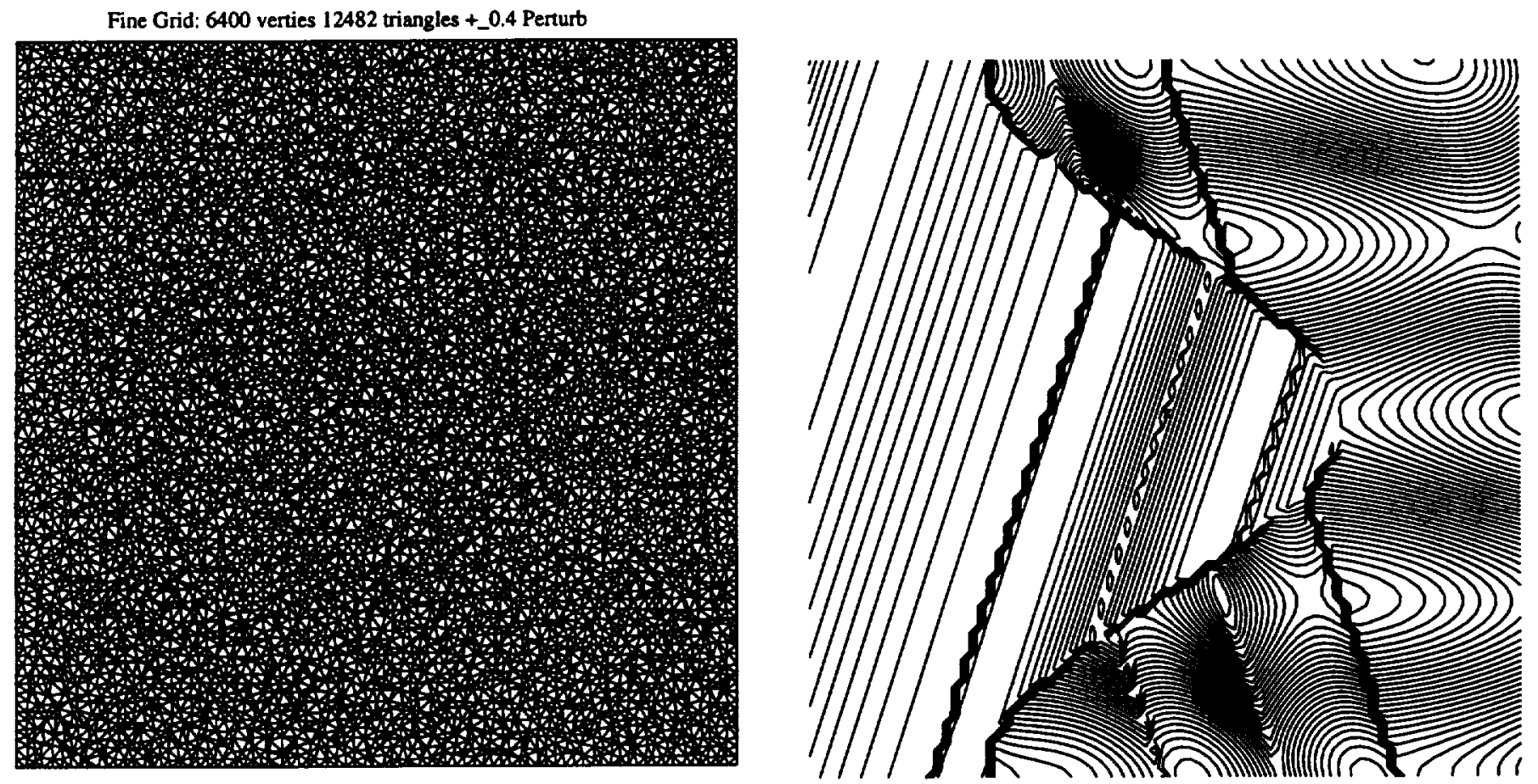

Figure 9: Unstructured Fine Grid. 6400 vertices and 12482 triangles. Generated by random perturbations of an $80 \mathrm{X}$ Figure 10: Exact solution on the fine grid 80 uniform structured grid.

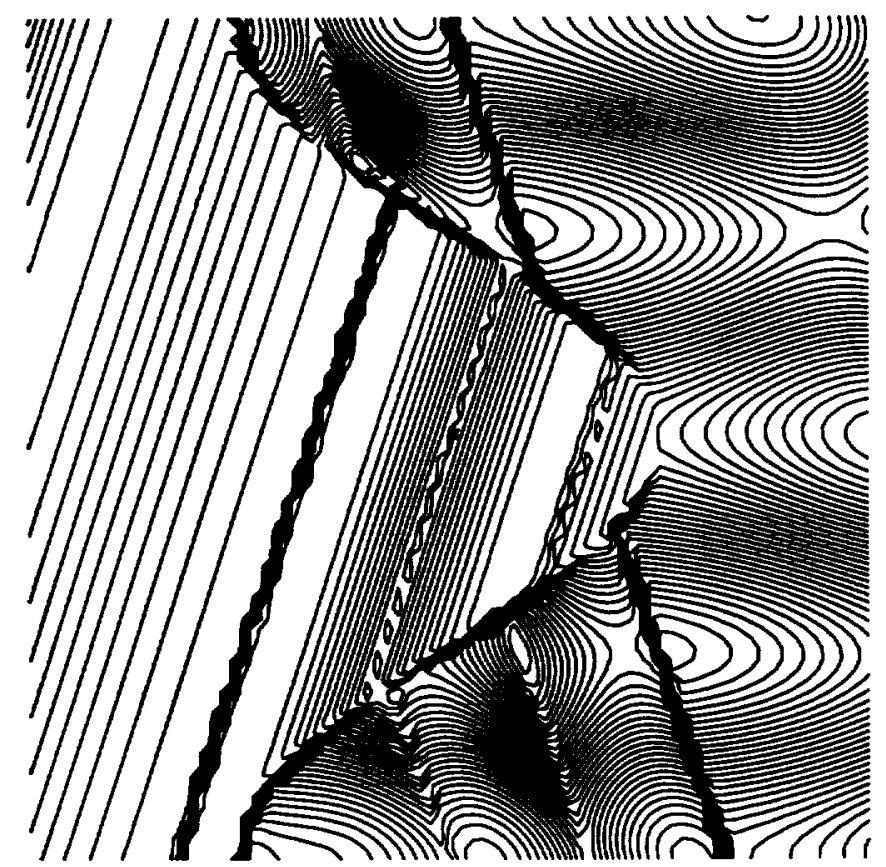

Figure 11: Fourth order ENOHC solution on the fine grid

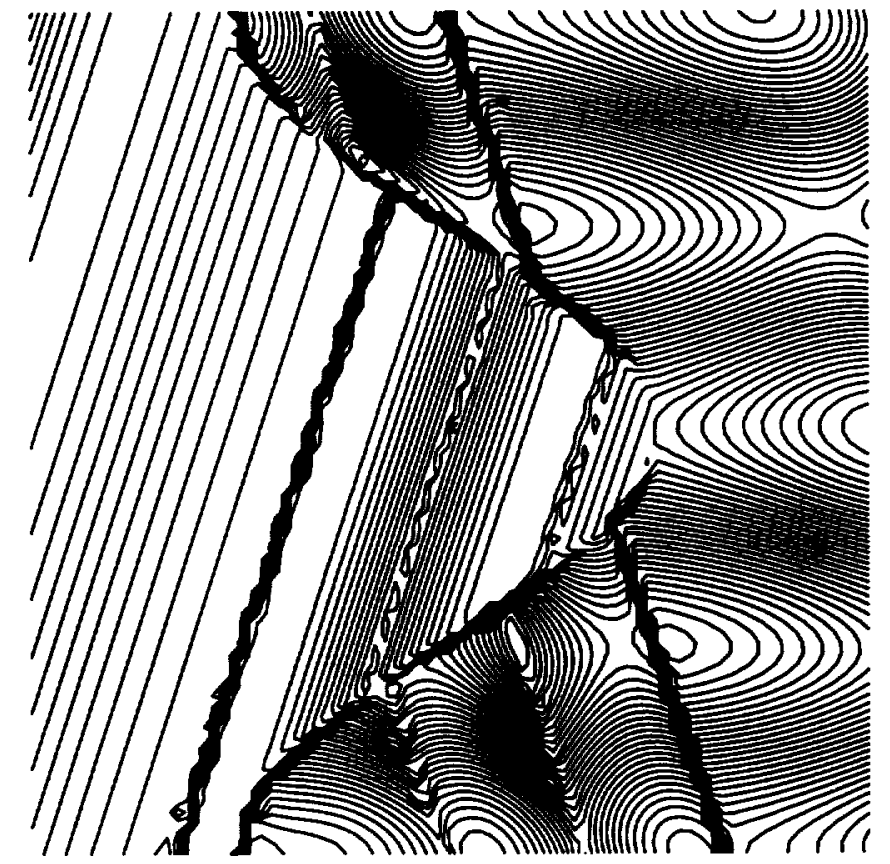

Figure 12: Fourth order ENOEX solution on the fine grid 

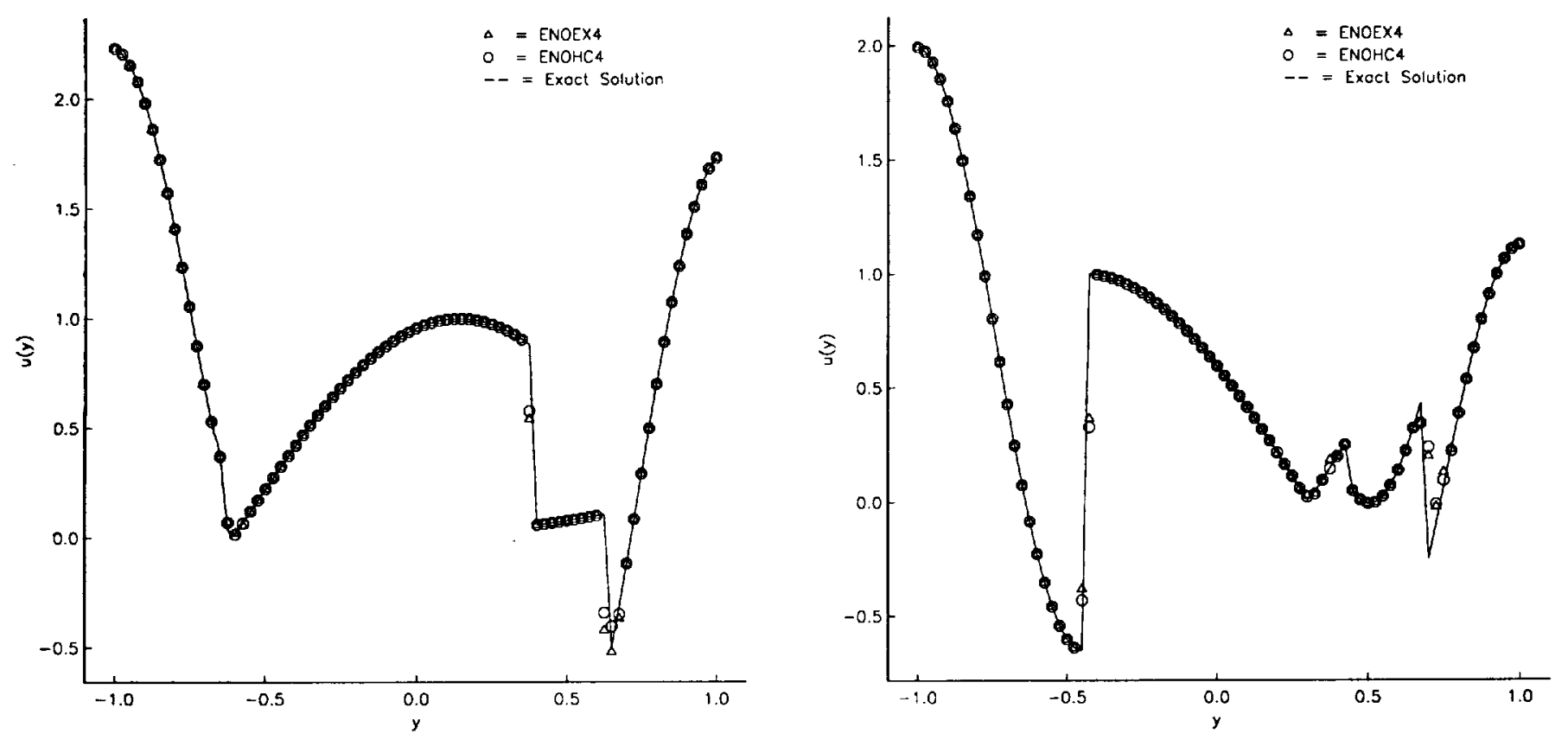

Figure 13: ENO Reconstructions on the line $x=-0.2$. Fine Figure 14: ENO Reconstructions on the line $x=+0.1$. Fine grid. grid.
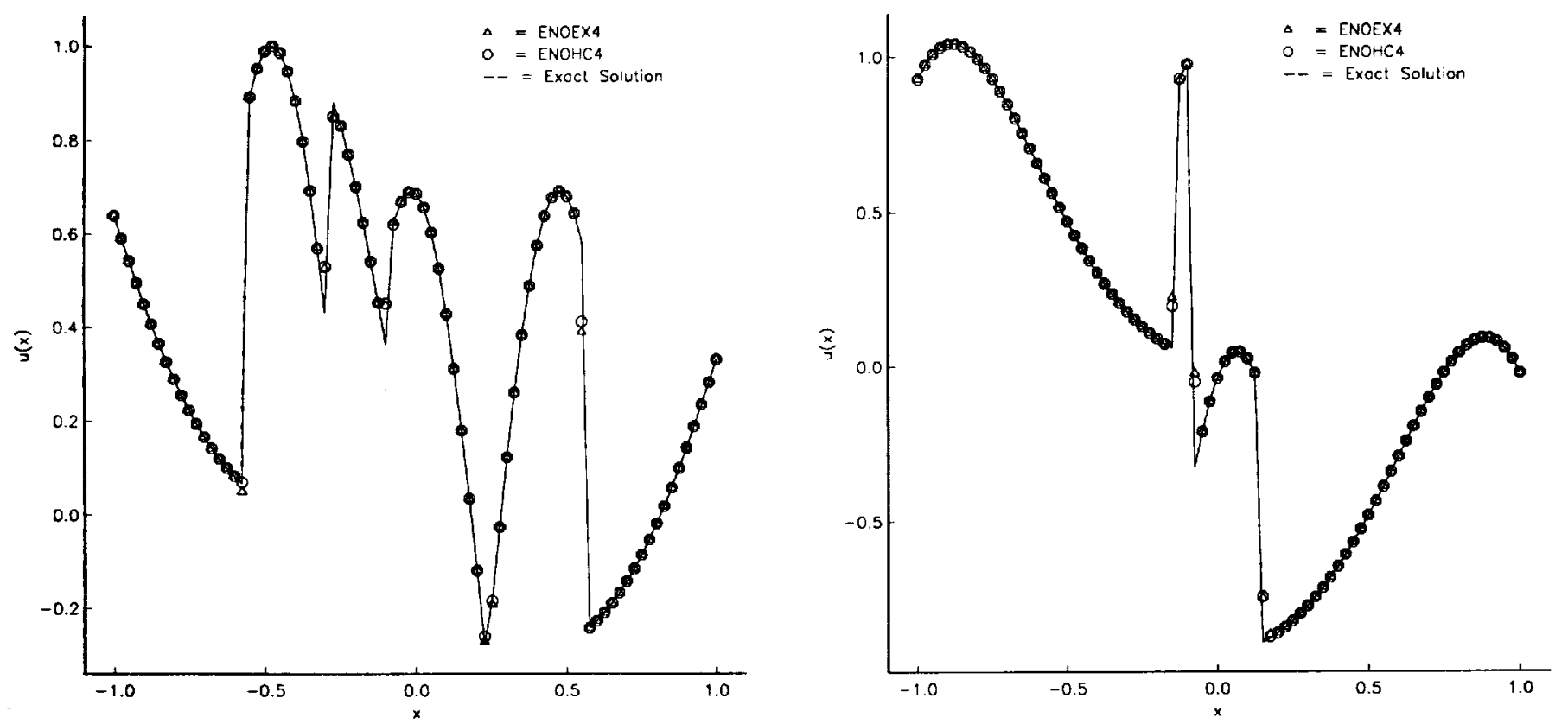

Figure 15: ENO Reconstructions on the line $y=-0.7$. Fine grid.

Figure 16: ENO Reconstructions on the line $y=0.55$. Fine grid. 
Public reporting burden for this collection of inlormation is estimated to average 1 hour per response, including the time for reviewing instructions. Bearching existing data sources, gathering and maintaining the data nooded, and compleing and reviewing the collection of intormation. Send comments regarding this burden estimate or any olher aspect of this Davis Highway, Suite 1204. Arlington, VA 22202-4302, and to the Office of Management and Budget, Papenwork Feduction Project (0704-0188), Washington, DC 20503.
1. AGENCY USE ONLY (Leave blank)
2. REPORT DATE
April 1995
3. REPORT TYPE AND DATES COVERED
Technical Memorandum

4. TITLE AND SUBTITLE

5. FUNDING NUMBERS

Essentially Nonoscillatory (ENO) Reconstructions via Extrapolation

6. AUTHOR(S)

WU-505-62-52

Ambady Suresh and Philip C.E. Jorgenson

\section{PERFORMING ORGANIZATION NAME(S) AND ADDRESS(ES)}

National Aeronautics and Space Administration

Lewis Research Center

Cleveland, Ohio 44135-3191
8. PERFORMING ORGANIZATION REPORT NUMBER

E-9607
9. SPONSORING/MONITORING AGENCY NAME(S) AND ADDRESS(ES)

National Aeronautics and Space Administration

Washington, D.C. 20546-0001
10. SPONSORING/MONITORING AGENCY REPORT NUMBER

NASA TM-106907

AIAA-95-0467

11. SUPPLEMENTARY NOTES

Prepared for the 33rd Aerospace Sciences Meeting and Exhibit sponsored by the American Institute of Aeronautics and Astronautics, Reno, Nevada, January 9-12, 1995. Ambady Suresh, NYMA, Inc., Engineering Services Division, 2001 Aerospace Parkway, Brook Park, Ohio 44142 (Work funded by NASA Contract NAS3-25266 with Sverdrup Technology, Inc., Lewis Research Center Group.) Philip C.E. Jorgenson, NASA Lewis Research Center. Responsible person, Philip C.E. Jorgenson, organization code 2610, (216) 433-5386.

12a. DISTRIBUTIONAVAILABILITY STATEMENT 12b. DISTRIBUTION CODE

Unclassified - Unlimited

Subject Categories 64,61 and 34

This publication is available from the NASA Center for Aerospace Information, (301) 621 -0390 .

13. ABSTRACT (Maximum 200 words)

In this paper, the algorithm for determining the stencil of a one-dimensional Essentially Nonoscillatory (ENO) reconstruction scheme on a uniform grid is reinterpreted as being based on extrapolation. This view leads to another extension of ENO reconstruction schemes to two-dimensional unstructured triangular meshes. The key idea here is to select several cells of the stencil in one step based on extrapolation, rather than one cell at a time. Numerical experiments confirm that the new scheme yields sharp nonoscillatory reconstructions and that it is about five times faster than previous schemes.

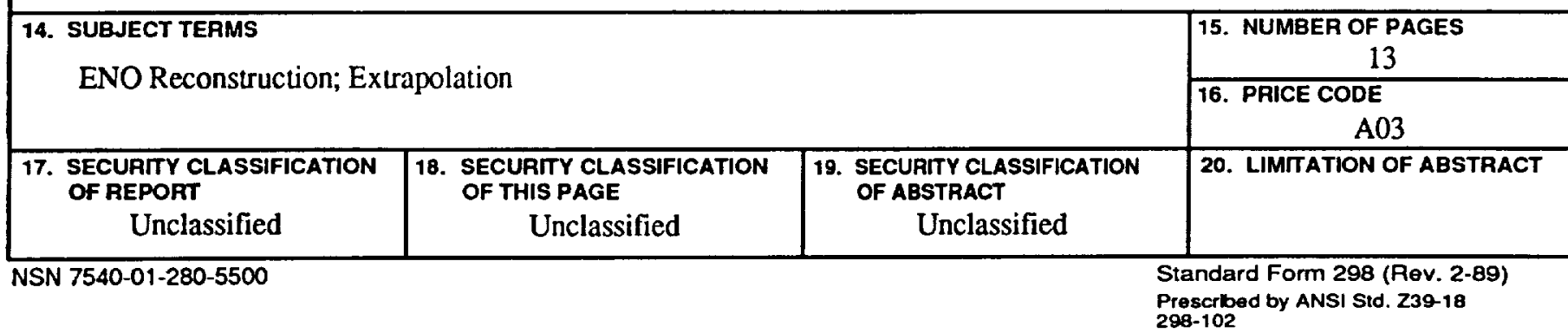

